\title{
Pronounced species divergence in corticospinal tract reorganization and functional recovery after lateralized spinal cord injury favors primates
}

\author{
Lucia Friedli, ${ }^{1}$ Ephron S. Rosenzweig, ${ }^{2}$ Quentin Barraud, ${ }^{1}$ Martin Schubert, ${ }^{3}$ Nadia Dominici, ${ }^{1,4}$

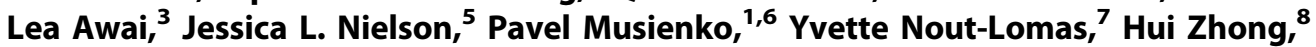 \\ Sharon Zdunowski, ${ }^{8}$ Roland R. Roy, ${ }^{8}$ Sarah C. Strand, ${ }^{9}$ Rubia van den Brand, ${ }^{1}$ \\ Leif A. Havton, ${ }^{10}$ Michael S. Beattie, ${ }^{11}$ Jacqueline C. Bresnahan, ${ }^{11}$ Erwan Bézard, ${ }^{12,13}$ \\ Jocelyne Bloch, ${ }^{14}$ V. Reggie Edgerton, ${ }^{8}$ Adam R. Ferguson, ${ }^{5}$ Armin Curt, ${ }^{3 *}$ \\ Mark H. Tuszynski, ${ }^{2,15}$ Grégoire Courtine ${ }^{1,14 \dagger}$
}

\begin{abstract}
Experimental and clinical studies suggest that primate species exhibit greater recovery after lateralized compared to symmetrical spinal cord injuries. Although this observation has major implications for designing clinical trials and translational therapies, advantages in recovery of nonhuman primates over other species have not been shown statistically to date, nor have the associated repair mechanisms been identified. We monitored recovery in more than $\mathbf{4 0 0}$ quadriplegic patients and found that functional gains increased with the laterality of spinal cord damage. Electrophysiological analyses suggested that corticospinal tract reorganization contributes to the greater recovery after lateralized compared with symmetrical injuries. To investigate underlying mechanisms, we modeled lateralized injuries in rats and monkeys using a lateral hemisection, and compared anatomical and functional outcomes with patients who suffered similar lesions. Standardized assessments revealed that monkeys and humans showed greater recovery of locomotion and hand function than did rats. Recovery correlated with the formation of corticospinal detour circuits below the injury, which were extensive in monkeys but nearly absent in rats. Our results uncover pronounced interspecies differences in the nature and extent of spinal cord repair mechanisms, likely resulting from fundamental differences in the anatomical and functional characteristics of the motor systems in primates versus rodents. Although rodents remain essential for advancing regenerative therapies, the unique response of the primate corticospinal tract after injury reemphasizes the importance of primate models for designing clinically relevant treatments.
\end{abstract}

\section{INTRODUCTION}

Despite the regenerative failure of severed axons after spinal cord injury (SCI), partial lesions of the human spinal cord are associated with spontaneous functional improvement during the first months after injury (1). Clinical observations suggest that the level of motor control asymmetry in the subacute phase of SCI influences the extent of functional recovery at chronic time points $(2,3)$. Patients with asymmetrical deficits, which are largely determined by the laterality of the spinal cord damage, generally exhibit greater functional gains compared to pa-

\footnotetext{
'Center for Neuroprosthetics and Brain Mind Institute, School of Life Sciences, Swiss Federal Institute of Technology (EPFL), 1015 Lausanne, Switzerland. ${ }^{2}$ Department of Neurosciences, University of California, San Diego, La Jolla, CA 92093-0662, USA. ${ }^{3}$ Spinal Cord Injury Center, Balgrist University Hospital, University of Zurich, 8008 Zurich, Switzerland. ${ }^{4}$ MOVE Research Institute Amsterdam, Faculty of Human Movement Sciences, VU University Amsterdam, 1081 BT Amsterdam, Netherlands. ${ }^{5}$ Department of Neurosurgery, University of California, San Francisco (UCSF), San Francisco, CA 94122, USA. 'Institute of Translational Biomedicine, St. Petersburg State University, St. Petersburg 199034, Russia. ${ }^{7}$ College of Veterinary Medicine and Biomedical Sciences, Colorado State University, Fort Collins, CO 80521, USA. ${ }^{8}$ Department of Integrative Biology and Physiology and Brain Research Center, University of California, Los Angeles (UCLA), Los Angeles, CA 900095-7246, USA. ${ }^{9}$ California National Primate Research Center, University of California, Davis, Davis, CA 95616-8542, USA. ${ }^{10}$ Department of Neurology, David Geffen School of Medicine, UCLA, Los Angeles, CA 90095-1769, USA. " ${ }^{11}$ Brain and Spinal Injury Center, UCSF, San Francisco, CA 94110, USA. ${ }^{12}$ Université de Bordeaux, Institut des Maladies Neurodégénératives, UMR 5293, F-33000 Bordeaux, France. ${ }^{13}$ CNRS, Institut des Maladies Neurodégénératives, UMR 5293, F-33000 Bordeaux, France. ${ }^{14}$ Clinical Neuroscience, University Hospital of Vaud (CHUV), 1011 Lausanne, Switzerland. ${ }^{15}$ Veterans Administration Medical Center, San Diego, CA 92161, USA. * Head of the European Multicenter Study about Spinal Cord Injury study group. tCorresponding author. E-mail: gregoire.courtine@epfl.ch
}

tients with more symmetrical deficits. For example, the most severe type of lateralized SCI, termed Brown-Séquard syndrome, is characterized by a complete acute loss of motor function on one side of the body, followed by substantial recovery of motor capacities in chronic stages (3). However, a direct relationship between the laterality of spinal cord damage and functional recovery has not been quantified in humans, and the mechanisms underlying this relationship remain unclear.

In primates, the corticospinal tract projects predominantly through the dorsolateral column and contains axons originating from both the left and right motor cortex (4). In contrast, the corticospinal tract is primarily located in the dorsal column in rodents, and these axons exclusively originate from the contralateral motor cortex. A substantial number of corticospinal axons decussate along the spinal cord midline in primates (4) but not in rodents. We previously showed that, after a lateral hemisection SCI in monkeys, these decussating corticospinal axons form detour circuits that reconnect the motor cortex with denervated segments (5). This reorganization of corticospinal projections below the injury correlated with spontaneous recovery of motor function (5). These results suggest that the specific architecture of the primate corticospinal tract and its unique response to injury, together with the essential contribution of these inputs to motor control in humans (6), may explain the superior functional recovery after lateralized compared to symmetrical injuries.

To address this hypothesis, we first compared the recovery profile of more than 400 quadriplegic individuals who suffered incomplete spinal cord damage covering the entire range of SCI laterality from 
perfectly symmetrical deficits to impairments restricted to one side of the body. Spontaneous restoration of function gradually increased with the degree of asymmetry. Second, we monitored the reorganization of corticospinal tract function in quadriplegic patients and modeled a lateralized SCI in rodents and monkeys to study anatomical remodeling of corticospinal projections in conjunction with electrophysiological and functional assessments. Our aim was to establish translational correlative structures (7) among anatomical, electrophysiological, and functional metrics across animal models and human patients. We found that the anatomical and functional properties of the corticospinal tract augment the potential for recovery after lateralized SCI in primates but not in rats, emphasizing the importance of primate models in translational research and for the development of spinal cord repair therapies.

\section{RESULTS}

\section{Functional recovery in humans correlates with SCI laterality} We conducted a repeated prospective assessment of functional recovery during 1 year after an incomplete cervical SCI in 437 patients. Only patients classified American Spinal Injury Association (ASIA)-C or ASIA-D with low motor scores were included in the analysis. To determine SCI laterality, we computed a laterality index (LI) based on the relative difference between left and right sensorimotor scores evaluated at 2 weeks after SCI. The index ranged from 0 (symmetric injury) to 1 (asymmetric injury affecting only one side of the body, as in BrownSéquard syndrome) (Fig. 1A and fig. S1A). More than $80 \%$ of analyzed patients reached values below 0.5 (Fig. 1B), indicating bilateral and symmetrical functional impairment. Only 4 of the 437 patients studied presented a pronounced Brown-Séquard syndrome with predominant unilateral motor deficits and crossed spinothalamic impairment. Magnetic resonance imaging (MRI) in these patients revealed white matter sparing restricted to one side of the spinal cord (Fig. 1A and fig. S1D). The LI had no significant influence on the severity of motor deficits at 2 weeks after SCI (Fig. 1C; $P=0.75$, ANOVA), indicating that the patients with symmetrical versus lateralized deficits exhibited similar impairments at early time points.

To assess whether SCI laterality influenced recovery, we computed the relative gains in left and right motor scores between early ( 2 weeks) and chronic (6 to 12 months) time points after injury. Improvements in motor function gradually increased with SCI laterality (Fig. 1D). Patients with an LI above 0.5 , who presented a noticeable asymmetry in motor performance at 2 weeks after injury, regained extensive bilateral motor function. These results demonstrate that spontaneous functional recovery increases with the degree of SCI laterality.

\section{Recovery of cortical access to motor pools below the lesion increases with SCI laterality in humans}

We monitored the recovery of motor responses in the tibialis anterior muscles in 34 quadriplegic patients after transcranial magnetic stimulation applied over the motor cortex (Fig. 2A). For patients with symmetric functional deficits, gains in motor response amplitude at chronic time points were generally proportional to the amplitude of the responses measured at early time points (Fig. 2, B and C). In contrast, patients with asymmetrical deficits ( $\mathrm{LI} \geq 0.5$ ) displayed a complete suppression of motor responses in the tibialis anterior muscle on the more affected side of the body at early time points (Fig. 2B). Substantial motor responses
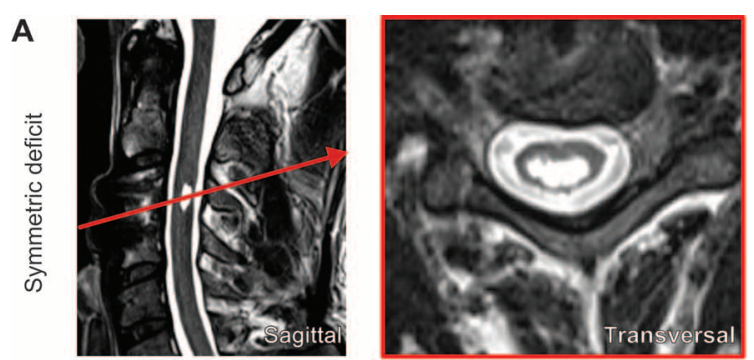

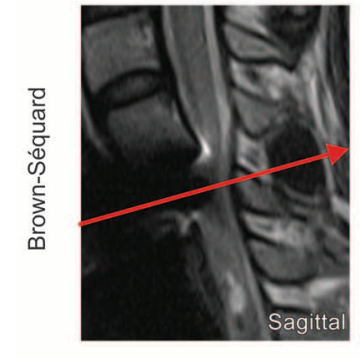

B
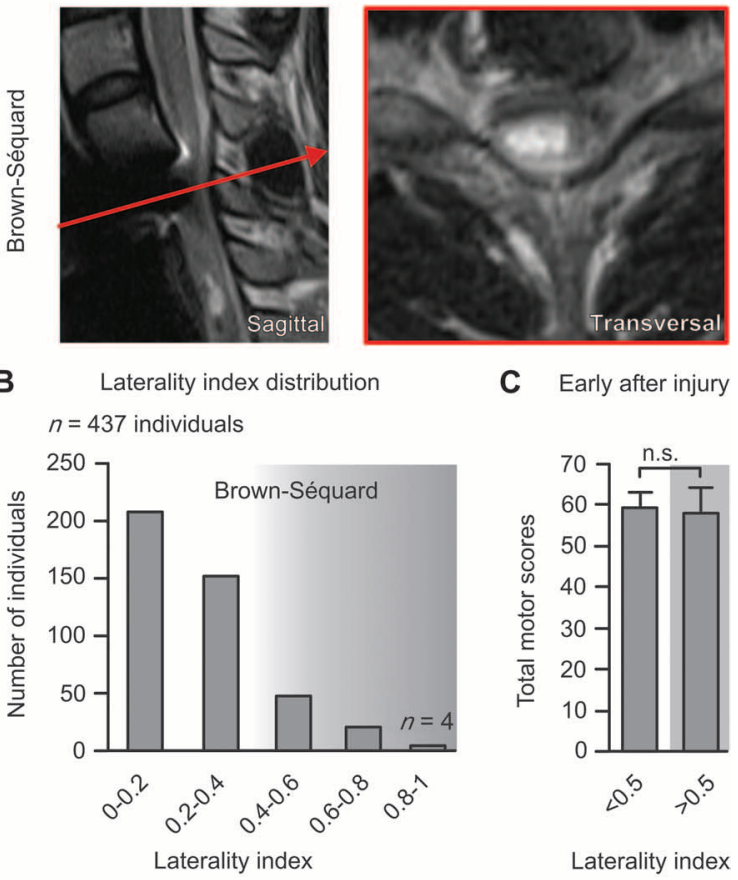

C Early after injury
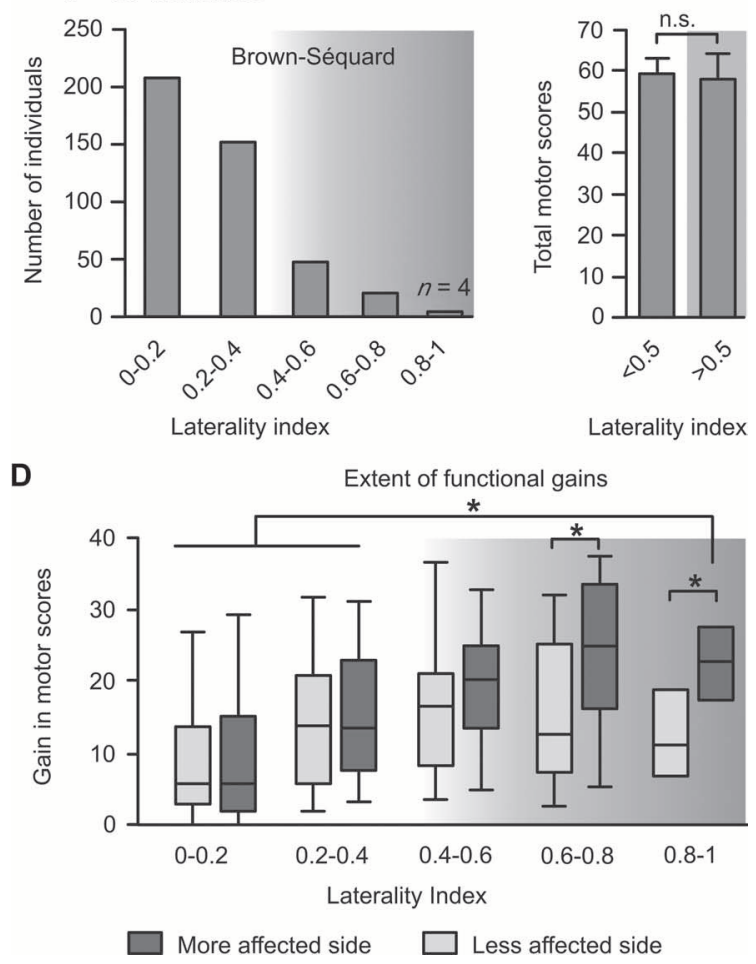

Fig. 1. Functional recovery correlates with $\mathrm{SCI}$ laterality in humans. (A) MR images representing a symmetrical and a lateralized SCI. (B) SCl laterality was measured as the relative difference between motor scores (ASIA) of the left and right sides, termed LI. The histogram shows the distribution of $\mathrm{LI}$ across 437 individuals with a cervical SCI (EMSCl database). The shaded area indicates individuals with asymmetric motor deficits. (C) Motor scores around 2 weeks after lesion for patients with $\mathrm{LI}<0.5$ and $\mathrm{LI} \geq 0.5$. (D) Gain in motor scores in the chronic compared to subacute stage of $\mathrm{SCl}$ for each range of LI. Horizontal bars indicate the median, and upper and lower bars correspond to the 75 and $25 \%$ percentile of data, respectively; error bars correspond to the 90 and $10 \%$ percentiles of the data $(n=437) .{ }^{*} P<0.05$, analysis of variance (ANOVA) followed by Turkey's post hoc test. n.s., not significant. 
A

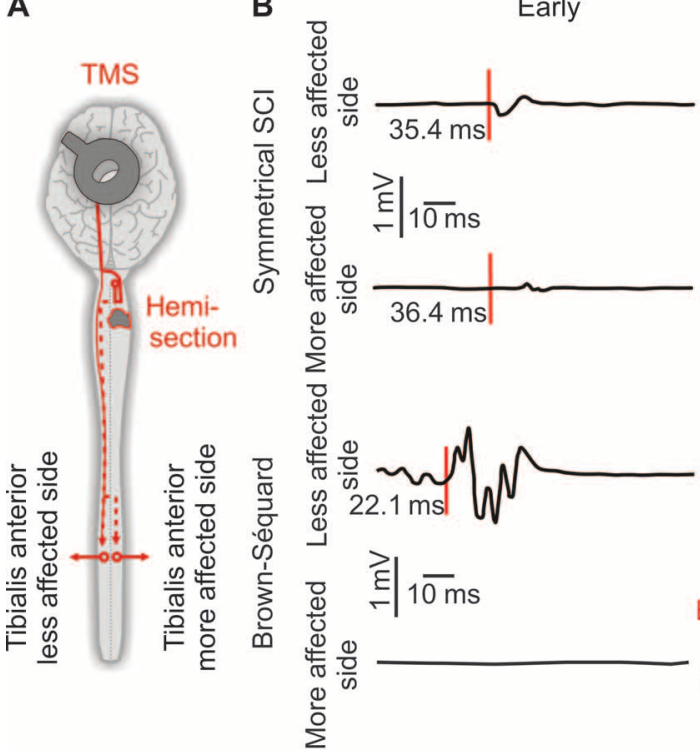

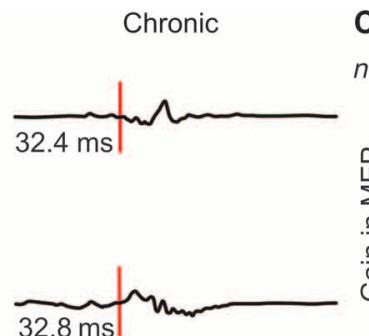

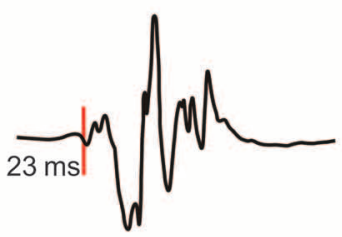

Expected latency

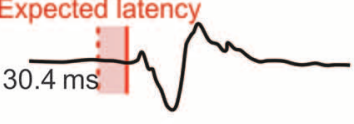

C Gain in motor response amplitude

$n=34$ More affected Less affected

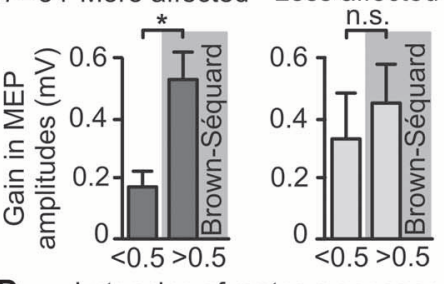

D Latencies of motor responses at chronic time points

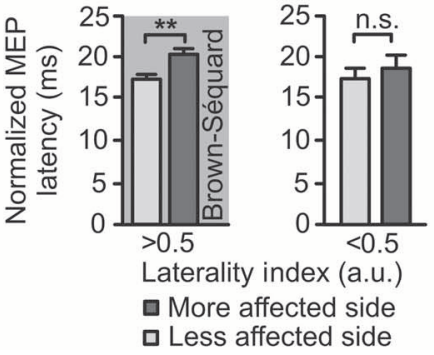

Fig. 2. Recovery of motor responses in leg muscles after motor cortex stimulation in quadriplegic patients. (A) Schematic overview of experiments. Motor responses were recorded in the left and right tibialis anterior muscles after transcranial magnetic stimulation (TMS) applied over the motor cortex. (B) Representative electromyographic (EMG) traces of left and right motor responses at early and chronic time points after SCI for a pa-

progressively reappeared in this muscle during recovery, which correlated with significantly larger gains in motor response amplitude in asymmetric SCI compared to patients with more symmetrical lesion at chronic time points (Fig. 2, B and C). In patients with asymmetric deficits, the latency of motor responses was significantly prolonged on the more affected side $(20.43 \pm 4.00 \mathrm{~ms})$ compared to the less affected side $(17.42 \pm 4.92 \mathrm{~ms})$ (Fig. 2D). No significant differences were found in the latencies of motor responses from left and right muscles in patients with more symmetrical spinal cord damage (LI <0.5).

\section{Recovery of locomotor function after lateralized $\mathrm{SCl}$ is greater in humans and monkeys than in rats}

To model a lateralized SCI, we placed a C7 lateral hemisection in monkeys and rats $(5,8)$. We performed standardized kinematics and muscle activity recordings to study the recovery profile of animal models compared to four human patients with distinct unilateral motor impairments in segments below the lesion at early time points (fig. S1D). Because hemiplegic patients could not step independently, we used the gait orthosis Lokomat to rhythmically move the legs along predefined foot trajectories (Fig. 3A). Despite stepping-like movements, ipsilesional ankle muscles remained quiescent, whereas contralesional muscles displayed alternating bursts of activity (Fig. 3A). At early time points, hemisected monkeys and rats essentially dragged their ipsilesional leg on the treadmill (Fig. 3, B and C). At chronic time points, all species tested showed extensive recovery in stepping ability: humans, monkeys, and rats regained plantar stepping on the ipsilesional side (Fig. 3, A to C). Specific features of locomotion, however, remained clearly impaired.

To measure these residual deficits, we submitted a larger number of gait parameters (table $\mathrm{S} 1$ ) to a $\mathrm{PC}$ analysis, which objectively quantified the features that are affected versus those nonaffected by injuries (9). tient with symmetrical versus asymmetrical deficits. (C) Mean gains in the amplitude of the motor responses on the less and more affected sides in patients with a low versus high LI. (D) Latency of motor responses from the less and more affected sides in patients with a low versus high LI. For (C) and $(D),{ }^{*} P<0.05,{ }^{* *} P<0.01$, unpaired Student's $t$ test. Data are means \pm SEM $(n=34)$. MEP, motor-evoked potential; a.u., arbitrary units.

Lateralized SCI altered the same variables in rats, monkeys, and humans (fig. S2, A to C). The rats, however, retained significantly more pronounced deficits at chronic time points compared to monkeys and humans (fig. S2D). For example, all species exhibited a significantly longer swing phase duration on the ipsilesional leg compared to the contralesional leg. Nevertheless, this asymmetry was markedly greater in rats (36\% longer on ipsilesional versus contralesional limb, $P<0.001$ ) compared to monkeys $(25 \%, P<0.05)$ and to patients $(3.1 \%, P<0.05)$ (ANOVA followed by Turkey's post hoc test).

To directly compare gait recovery between rats, monkeys, and humans, we normalized all computed parameters to intact values for each species and applied PC analysis on all the normalized data sets simultaneously (Fig. 3D). Gait clusters related to chronic time points occupied significantly more distant locations to intact gait clusters in rats compared to monkeys and humans (Fig. 3E). This analysis demonstrates that monkeys and humans show a greater recovery of gait compared to rats after a lateralized SCI (movie S1).

Differences in gait recovery were particularly striking during more challenging conditions, such as traversing a horizontal ladder. The four patients executed this task with $100 \%$ success (Fig. 4A). In contrast, rats failed to position their ipsilesional hindpaw onto the rungs of the ladder (Fig. 4B). This failure was not due to deficits in forelimb placement because rodents with a thoracic hemisection also display permanent loss of hindpaw placements during locomotion along ladders (9). The patients performed well on the horizontal ladder despite the use of distinct gait strategies and slight postural instability compared to healthy subjects (Fig. 4, C and D, and movie S1). Notwithstanding differences in the execution of bipedal versus quadrupedal locomotion along ladders, there was a strong disparity between rats and humans in the recovery of leg motor skills. For safety reasons, monkeys were not tested on this task. 

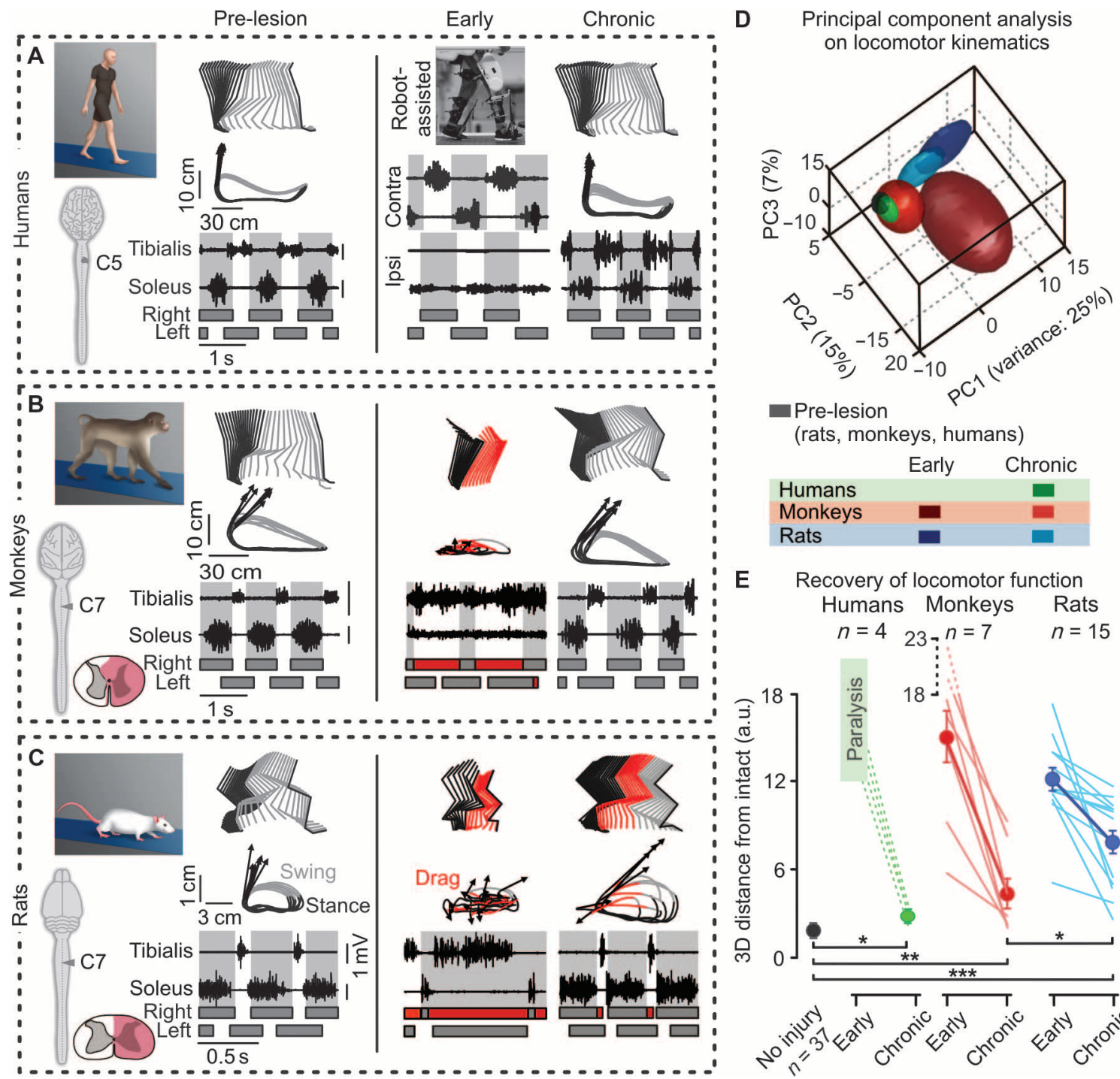

- Pre-lesion

(rats, monkeys, humans)

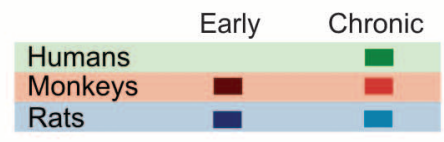

E Recovery of locomotor function

E Humans Monkeys Rats

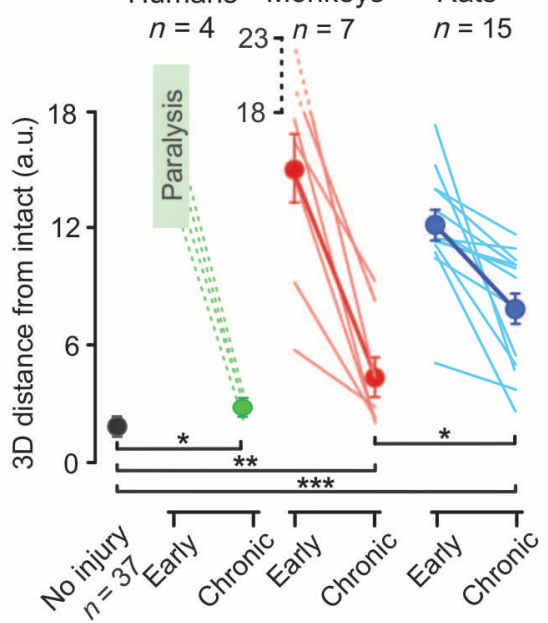

Fig. 3. Humans and monkeys show greater recovery of locomotion compared to rats after lateralized SCI. (A to C) Decomposition of lower limb motion during stance and swing together with successive limb endpoint trajectories during locomotion on a treadmill. Representative data are shown for a human patient $(A)$, monkey $(B)$, and rat $(C)$ before the injury (or healthy) and at early and chronic time points after $\mathrm{SCl}$. The vectors indicate the direction and amplitude of foot acceleration at swing onset. The EMG activity of the soleus and tibialis anterior muscles is shown at the bottom. The shared areas indicate the duration of the stance phases.

\section{Hand function recovery is more extensive in monkeys and humans than in rats}

Rodents and primates exhibit different upper limb motor skills. To establish reasonable comparisons across species, we implemented a reach-for-food task that has been shown to involve similar hand control strategies in rats, monkeys, and humans (10). The size of the retrieved food was scaled for each species to fit within the palm of the hand. Kinematics and muscle activity recordings revealed that, overall, noninjured rats, monkeys, and humans used a comparable strategy to retrieve food items with the hand (Fig. 5, A to C, and fig. S3). However, the performance was significantly worse in rats compared to monkeys
At early time points, hemiplegic patients were recorded in the gait orthosis Lokomat. (D) Principal component (PC) analysis was applied on dimensionless parameters $(n=101)$, characterizing gait patterns of rats, monkeys, and humans. Least-squares spheres are traced to help visualize gait clusters and thus emphasize time- and species-dependent gait recovery. (E) Individual (lines) and mean three-dimensional (3D) distances between noninjured gait clusters and those measured at the early and late time points. ${ }^{*} P<$ $0.05,{ }^{* *} P<0.01,{ }^{* * *} P<0.001$, ANOVA. Data are means \pm SEM ( $n$ indicated in figure).

and humans (Fig. 5D). The patients who had completely lost ipsilesional hand function at early time points performed the reach-for-food task with $100 \%$ success at the chronic time point (Fig. 5, A and D, and movie S2).

In both monkeys and rats, the hemisection resulted in loss of detectable function and muscle activity in the ipsilesional hand immediately after injury (main effect of injury; $P<0.001$, ANOVA; Fig. 5 , B and C). Over time, hemisected monkeys regained the capacity to recruit extensor and flexor digit muscles reciprocally (Fig. 5B and fig. S3B), which paralleled extensive recovery of object retrieval (Fig. 5D). The improved performance in monkeys often was accompanied by a change in the reward-retrieval strategy, including grasping between the fingers and 

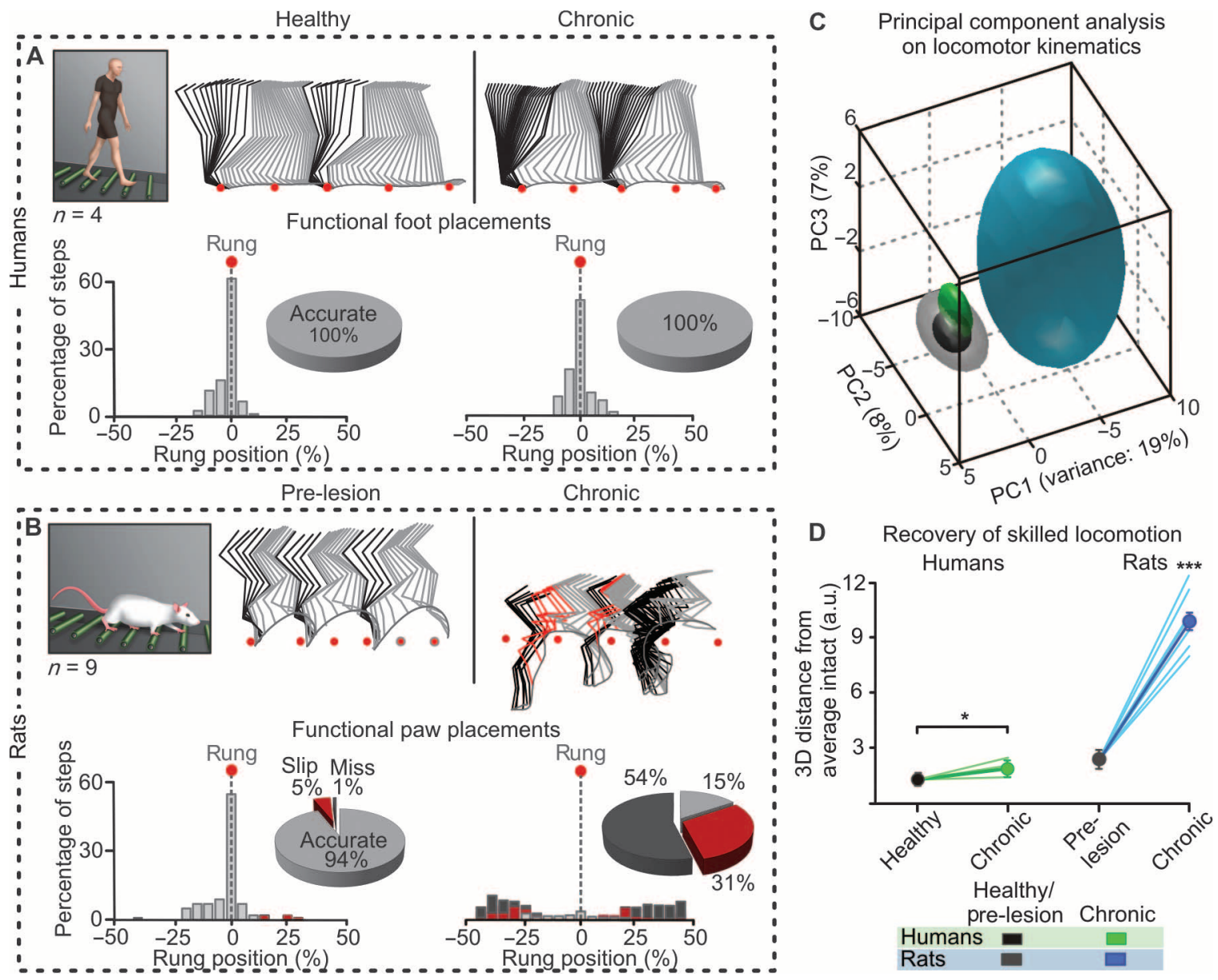

Fig. 4. Humans, but not rats, recover the ability to traverse a horizontal ladder after lateralized SCI. (A and B) Decomposition of lower limb motion during locomotion along the equally spaced rungs of a horizontal ladder. Foot placement was measured as the relative positioning of the ipsilesional foot with respect to two successive rung positions (red dots). The number of occurrences per $5 \%$ bin is reported together with the percentage of accu- rate, slipped, and missed placements. (C) PC analysis performed using the same conventions as in Fig. 3D: 101 kinematic parameters measured for the ipsilesional leg before the injury (or healthy) and at the chronic stage of SCl. Individual (lines) and averaged 3D distance between noninjured data points and those measured at late time points. ${ }^{* *} P<0.001$, ANOVA. Data are means \pm SEM ( $n=4$ humans, 15 rats). the palm of the hand in a subset of monkeys (11). Despite repeated training with motivational cues, none of the 15 rats tested regained the ability to retrieve a single food reward with the injured paw $(P<0.001$, ANOVA) (Fig. 5, C and D, and movie S2). Rats produced highly variable paw trajectories and disorganized muscle activation patterns of muscles (Fig. 5C and fig. S3C). Although disparities in cognitive and motor skills may partly explain the different motor recovery of rodents versus primates, the extent of this discrepancy favors the existence of distinct mechanisms supporting greater recovery in primates compared to rats.

\section{Monkeys show greater reorganization of corticospinal tract below the injury than rats}

We next investigated the reorganization of axonal projections from the corticospinal tracts in both monkeys and rats. Corticospinal fibers were labeled with injections of anterograde tracers in the left and right motor cortex (Fig. 6, A and B). We compared corticospinal projection patterns in rats and monkeys terminated at early ( $n=8$ rats and 3 monkeys) or chronic ( $n=7$ rats and 9 monkeys) time points after SCI. Four of the nine monkeys were in previous reports $(5,11,12)$ and underwent new analyses for this study.

Intact monkeys showed extensive bilateral corticospinal tract projections (fig. S1B) (4). Most corticospinal tract fibers projected through the dorsolateral columns. Consequently, the lateral hemisection in monkeys spared a substantial number of corticospinal axons originating from both the left and right motor cortex (Fig. 6C). In rats, the vast majority of corticospinal fibers decussated at the level of pyramids, extended rostrocaudally in the dorsal column (fig. S1C), and projected contralaterally in the dorsal and intermediate laminae of the spinal segments (Fig. 6D). Therefore, the lateral hemisection interrupted nearly all axonal projections from the contralesional motor cortex in the rats (Fig. 6D). A very small subset of axons projecting ipsilaterally through the ventral funiculus remained intact in rats (Fig. 6F).

We first analyzed the reorganization of corticospinal fibers originating from the motor cortex contralateral to the hemisection. Monkeys exhibited a significant increase in the number of axons decussating at the spinal cord midline below the lesion at chronic compared to early time points (Fig. 6, E and G), which was absent in hemisected rats (Fig. 6, 

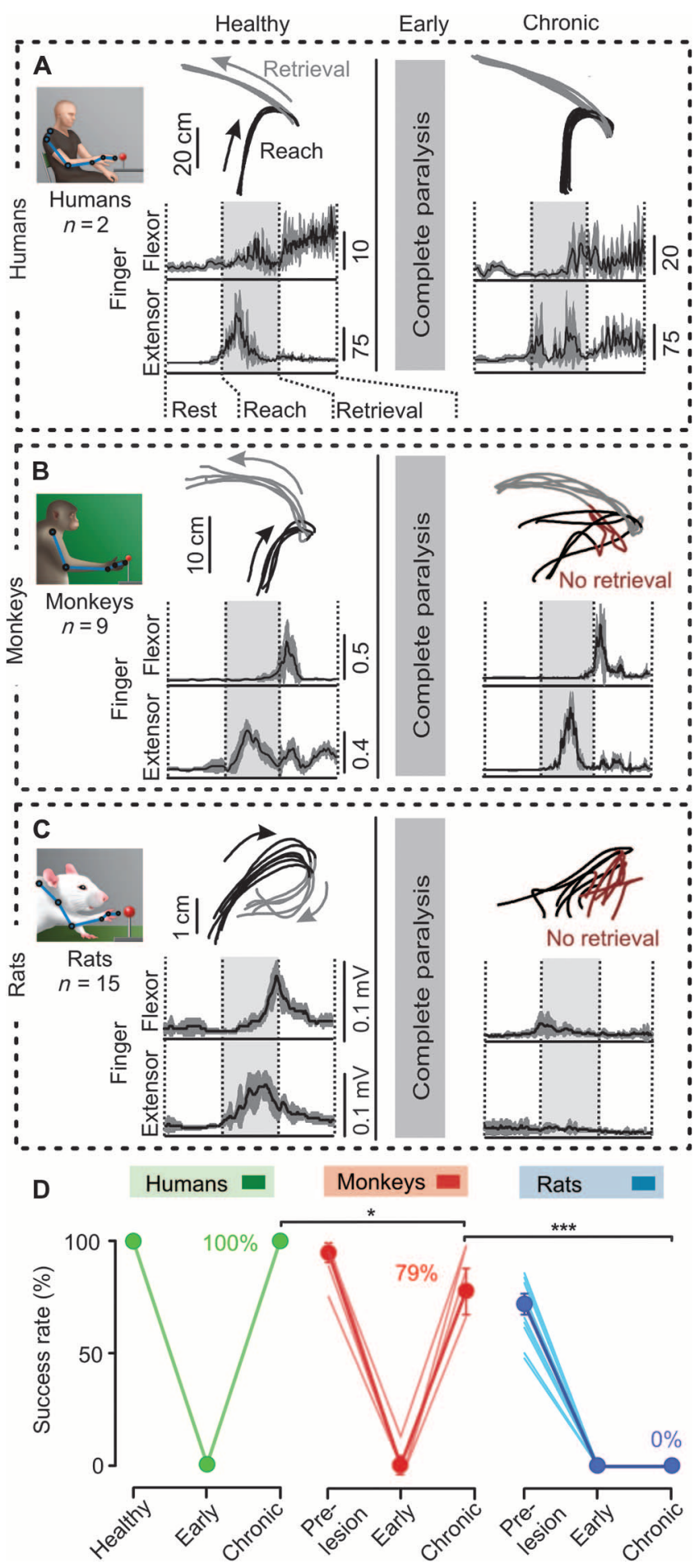

Fig. 5. Monkeys and humans show greater recovery of hand function compared to rats. (A to $\mathbf{C}$ ) Upper limb endpoint trajectories during reach and retrieval. Failures of item retrieval are displayed in dark red, seen after $\mathrm{SCl}$ in rats and monkeys but not in humans. Averaged EMG activity ( \pm SEM in gray) of the extensor digitorum communis and flexor digitorum muscles is shown during successive retrievals before the injury (or healthy) and at chronic time points after $\mathrm{SCl}$ in humans (A), monkeys (B), and rats (C). (D) Percent of successful object retrieval for each subject. ${ }^{*} P<0.05,{ }^{* * *} P<$ 0.001, ANOVA. Data are means \pm SEM $[n$ indicated in $(A)$ to $(C)]$.
$\mathrm{F}$ and $\mathrm{H}$ ). These axons displayed an increased density within spinal segments below the injury, in particular, into the denervated ventral horns that contain motor pools innervating hand muscles (early versus chronic; $P<0.05$, ANOVA followed by Turkey's post hoc test; Fig. 6, C and G). This sprouting reconstituted a large proportion of the corticospinal fiber density observed in intact monkeys, which contrasted with the near-complete depletion of corticospinal fibers observed in hemisected rats (Fig. 6, D and $\mathrm{H}$ ).

Fiber reconstructions revealed that corticospinal axons below the injury originated from the dorsolateral column in monkeys (Fig. 6I) and the ventral component of the corticospinal tract in rats (Fig. 6J). These fibers, however, were sporadic in rats. Corticospinal axons below the injury established synapses, because they colocalized with synaptophysin (Fig. 6, K and L). In hemisected monkeys, corticospinal fibers decussating the spinal cord midline below the injury established synaptic contacts with neurons projecting to lumbar segments (fig. S4). Analysis of corticospinal axon density at C5 and C6, above the lesion, also revealed a significant increase in density at chronic compared to early time points in rats, but only a trend in monkeys (fig. S5). In both rats and monkeys, axons originating from the right motor cortex decussated at the level of the pyramids and then recrossed the spinal cord midline below the hemisection. The density of these fibers was significantly increased in denervated segments for rats and monkeys terminated at chronic compared to early time points $(P<0.05$, ANOVA; fig. S6). Remodeling of spared axons did not occur in all descending projection neurons: the density or distribution of serotoninergic axons in cervical segments below the injury remained unchanged in rats and monkeys (fig. S7).

These anatomical evaluations reveal that both rats and monkeys exhibit multilevel reorganization of bilateral corticospinal projections after hemisection. Only monkeys, however, showed growth of corticospinal fibers across the spinal cord midline and extensive sprouting of these fibers into denervated spinal segments below the injury.

\section{Recovery of cortical access to leg motor pools is greater in humans than in rats}

The poor reorganization of corticospinal fibers below the hemisection suggested that, in contrast to humans, rats would not regain motor responses in leg muscles after motor cortex stimulation. To test this hypothesis, we delivered electrical stimulations in awake rats through a chronically implanted electrode over the motor cortex and recorded motor responses in ankle flexor muscles (fig. S8A) (13). In intact rats, motor cortex stimulation evoked short latency motor responses that likely reflect the cortical recruitment of fast-conducting brainstem descending pathways (fig. S8B) (14). The hemisection nearly permanently suppressed these motor responses in ankle flexor muscles ipsilateral to the lesion (fig. S8C).

\section{Corticospinal tract reorganization correlates with functional recovery}

Last, we sought to evaluate whether functional recovery correlated with specific anatomical and electrophysiological features. We conducted the analyses using the subsets of variables that were collected from rats and monkeys or from rats and humans, allowing direct crossspecies comparisons. We first calculated the correlation matrix between all variables, which revealed robust correlations between anatomical, electrophysiological, and functional metrics across species (Fig. 7A). We then applied a nonlinear PC analysis (NLPCA) onto these variables 


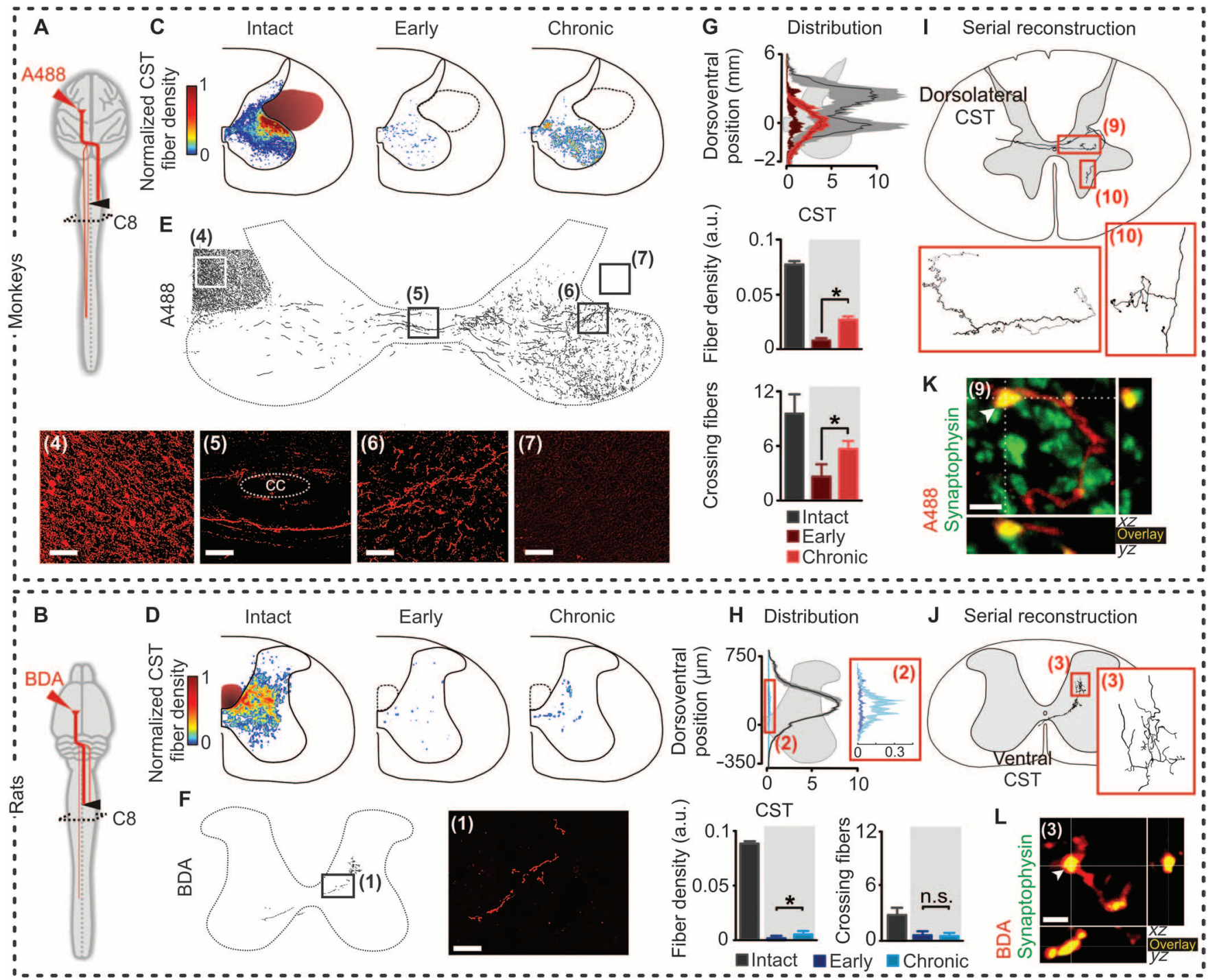

Fig. 6. Monkeys show greater reorganization of corticospinal tract (CST) fibers compared to rats. Corticospinal projection patterns were measured in rats and monkeys terminated at early ( $n=8$ rats and 3 monkeys) or chronic ( $n=7$ rats and 9 monkeys) time points after $\mathrm{SCl}$. (A and B) Diagrams illustrating anatomical experiments. (C to F) Heat maps and representative images and reconstructions taken from boxed and numbered areas, illustrating sprouting of corticospinal axons in spinal segments below the lesion (CC, central canal). Scale bars, $100 \mu \mathrm{m}$ for monkeys and $40 \mu \mathrm{m}$ (insets: $10 \mu \mathrm{m}$ ) for rats. (G and $\mathbf{H}$ ) Fiber density distribution, ipsilesional corticospinal axon density at $\mathrm{C} 8$, and number of

(15). NLPCA uncovers multidimensional correlative structures between isolated metrics of different scales, characterizing various aspects of a syndrome, and ranking individual subjects within this syndromic space $(7,16)$.

PC1 and PC2 explained a considerable amount of variance in the functional electrophysiological and anatomical variables (Fig. 7B). We then extracted the PC loadings, which corresponded to correlations between the variables and PC1 or PC2, and represented these values in clusters of correlated variables (Fig. 7B). The rat versus human anal- corticospinal fibers crossing the spinal cord midline per analyzed section at $\mathrm{C} 8$ in intact animals and at early and chronic time points after $\mathrm{SCl}$. ${ }^{*} P<$ 0.05 , ANOVA. Data are means \pm SEM ( $n=8$ monkeys; 9 rats for chronic subjects). (I and J) Serial reconstruction of a single corticospinal axon. ( $\mathbf{K}$ and $\mathbf{L}$ ) Representative confocal images of respective area in (I) or (J), showing 3D colocalization of dextran-conjugated Alexa Fluor 488 (D-A488) and synaptophysin in monkeys, and biotin dextran amine (BDA) and synaptophysin in rats, demonstrating the presence of synaptic terminals onto corticospinal fibers below the hemisection. Scale bars, 2 and $4 \mu \mathrm{m}$ for monkeys and rats, respectively.

ysis revealed robust correlations between the recovery of motor cortex access to motoneurons innervating leg muscles and recovery of basic locomotion and reaching, in both rats and humans. For rat versus monkey, the degree of multilevel reorganization of the left and right corticospinal tract correlated with increased recovery of locomotion, and even more extensively, hand function, in both species. Analysis of individual PC scores, however, revealed that these mechanisms of recovery were significantly more efficient in monkeys (PC1) and humans (PC2) than in rats $(P<0.05$, ANOVA) (Fig. $7 \mathrm{C})$. 


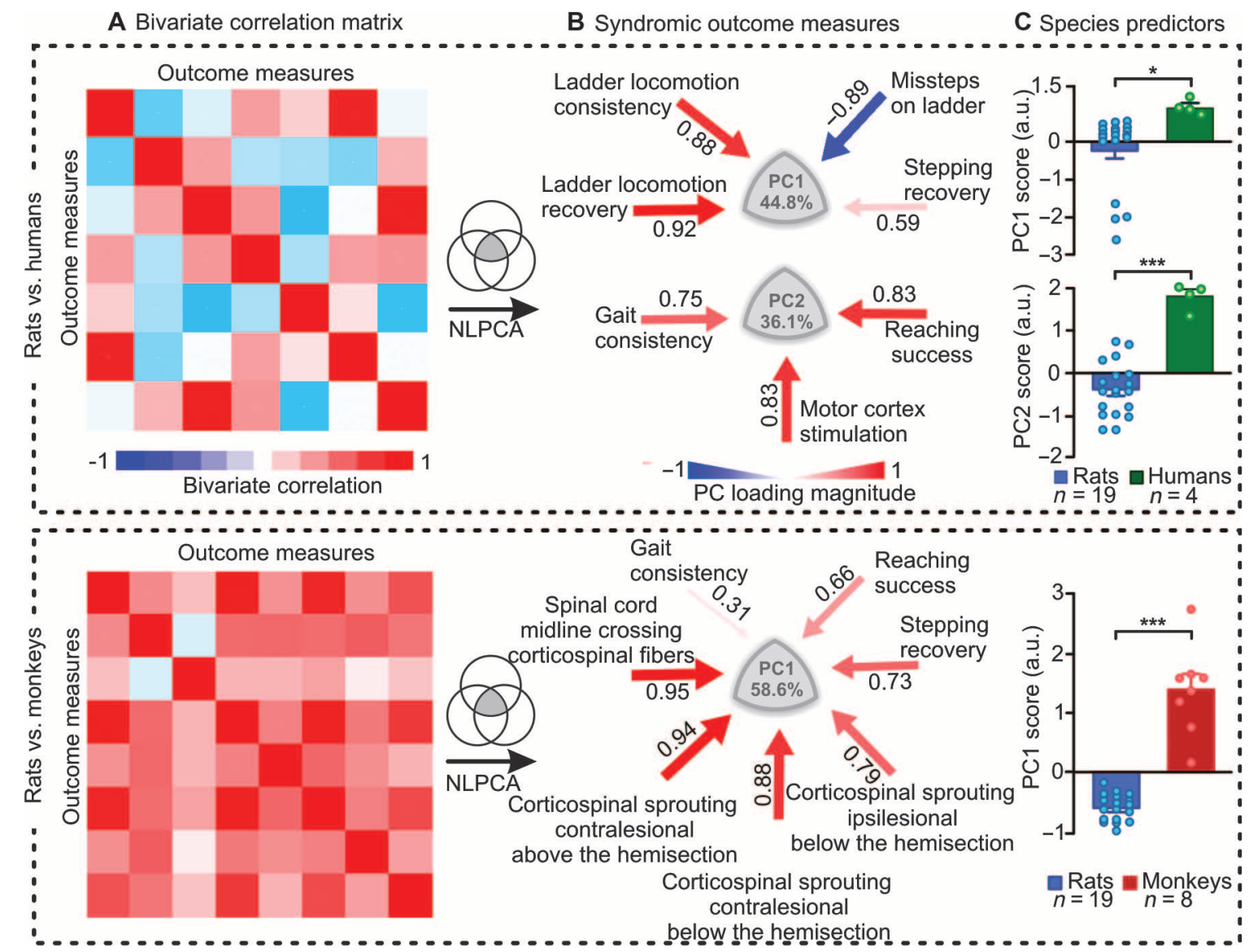

Fig. 7. Syndromic analysis linking reorganization of corticospinal tract function and functional recovery. We used subsets of variables that were collected from rats and monkeys or from rats and humans for direct cross-species comparisons. (A) Bivariate correlation matrix showing robust correlations between anatomical and functional parameters. (B) An NLPCA was applied on all the parameters measured in rats and humans and in rats and monkeys. The data variance explained by PC1 and
PC2 is reported. Color- and size-coded arrows indicate the direction and correlation (red, positive; blue, negative) between the parameters and each PC. Ipsilesional and contralesional refer to the origin of the corticospinal tract. (C) Mean scores on PC1 for both analyses and on PC2 for human versus rat. Each dot represents an individual subject. ${ }^{* *} P<0.001$, ${ }^{* *} P<0.01$, unpaired two-tailed $t$ tests. Data are means \pm SEM ( $n$ indicated in figure).

\section{DISCUSSION}

Our study of more than 400 quadriplegic patients and both rodent and primate injury models demonstrates that anatomical and functional evolution of the corticospinal tract has produced fundamental interspecies differences in the nature and extent of spinal cord repair mechanisms. The corticospinal tract is not necessary for the execution of noncomplex movement in lower mammals (17). In monkeys, the selective ablation of this pathway induces permanent deficits in skilled motor control but has minimal impact on locomotion $(18,19)$. Indirect evidence suggests that humans rely heavily on corticospinal input for the production of voluntary movement (20), although the contribution of this tract to locomotion remains debated (21). This increasingly important role of corticospinal tract function in motor control correlates with multifaceted adaptations in the anatomical properties of this pathway during primate evolution (22). Our results show that these adaptations provide an important advantage for recovery after injury. We discuss these findings on interspecies differences in SCI and repair with an emphasis on implications for clinical trial design, therapeutic development, and translational medicine.
Our epidemiologic study confirms that individuals with a pronounced Brown-Séquard syndrome are rare. Nonetheless, we demonstrate that patients with partial SCI cover a broad spectrum of laterality in spinal cord damage and that the extent of laterality significantly influences recovery. These results stress the importance of integrating an LI in predictive models of recovery after SCI. In turn, the degree of laterality should be carefully considered for the selection of homogeneous patient cohorts for clinical trials, because our results show that this factor will influence functional outcomes at extended time points.

The superior recovery of patients with lateralized versus symmetrical injuries offered a unique experimental opportunity to investigate some of the key mechanisms underlying spontaneous improvement in function after SCI. What are the mechanisms most likely to account for the greater recovery after lateralized compared to symmetrical injuries in humans? Previous work in rodents demonstrated that this recovery relies on the establishment of detour circuits reconnecting brainstem and intraspinal descending pathways to denervated motor circuits below the lesion $(9,13,23-27)$. Increased collateralization of cortical projections into the brainstem and spinal cord above the injury 
allows the motor cortex to take advantage of these detour circuits to access spinal circuits below the injury $(8,13,23,28)$. After a lateral hemisection SCI in rodents, this circuit rearrangement restores extensive basic locomotor capacities but is insufficient to mediate skilled movement (9). The same mechanisms likely participate in the motor recovery of humans with lateralized injuries. However, our results provide evidence that in primates, contrary to observations in rats, spared corticospinal fibers also establish detour circuits that form a bridge across the hemisection, and that this reorganization is closely related to improvement in fine motor control capacities.

We derive these conclusions from the combination of functional, electrophysiological, and anatomical outcomes across humans and animal models. First, we report relationships between the functional gains in patients with lateralized deficits and the recovery of access of the motor cortex to denervated motoneurons below the injury. Patients with symmetrical damage did not exhibit similar gains, indicating that this recovery was due to a specific reorganization of the corticospinal tract rather than adaptations in spinal circuits (29). The latency of the regained motor responses increased on the hemisected side, which was consistent with the formation of compensatory detour circuits that restore cortical influence over motoneurons innervating leg muscles below the injury.

Second, we modeled a lateralized injury in rats and monkeys to study the anatomical reorganization of both corticospinal tracts, which have undergone profound anatomical adaptations during evolution (22): The corticospinal tract migrated from the dorsal column to the dorsolateral funiculi, which correlated with an increase in the relative number of axons (30); in addition, the pattern of corticospinal projections became increasingly bilateral, with a substantial number of axons decussating extensively across the spinal cord midline (4); finally, the appearance of direct cortical projections onto motoneurons correlated with the emergence of a precision grip between the thumb and the index fingers $(6,31)$. Anatomical evaluations showed that these adaptations confer superior advantages for recovery after SCI in primates compared to rodents, especially after lateralized damage. Owing to their course in the dorsolateral column, the lateral hemisection spares numerous corticospinal fibers originating from both motor cortexes in primates. These fibers decussate across the spinal cord midline $(4,5)$ and terminate bilaterally, including in the gray matter below the injury. The lateral hemisection triggered a growth of these axons across the spinal cord midline, and an extensive sprouting of spared and newly formed fibers into spinal territories containing both denervated hand motoneurons and neurons projecting to lumbar segments. Thus, these corticospinal detour circuits reestablished a direct cortical access to the gray matter below the injury. In rats, the lateral hemisection spared a small subset of ipsilaterally projecting fibers located in the ventral funiculus, which contribute to recovery after an incomplete SCI (32). The density of these undamaged corticospinal tract axons increased after the lateral hemisection. However, our multidimensional correlation analyses suggest that the relevance of this anatomical reorganization for functional recovery is modest in rats.

Post-mortem anatomical analyses (31), imaging (33), and electrophysiological recordings $(34,35)$ have provided indirect evidence that sprouting of spared corticospinal fibers contributes to functional recovery after SCI in humans. Our multivariate analyses established robust correlations between bilateral corticospinal tract reorganization and functional recovery in humans, monkeys, and, albeit weakly, rats. As expected, correlations were more pronounced for skilled motor tasks than for locomotion. These results support previous studies that proposed corticospinal tract reorganization as a fundamental mechanism of recovery after SCI in mammals $(5,29)$. However, owing to the bilateral projection patterns of corticospinal axons in higher mammals, and probably, the more advanced contribution of these inputs to motor control and learning $(22,36)$, this mechanism is more efficacious in monkeys and humans compared to rodents. The greater plasticity and functional contribution of the primate corticospinal tract may provide additional degrees of freedom for guiding and using newly formed circuits in the spinal cord.

Our kinematics and electromyography methodologies across multiple behavioral paradigms demonstrate that this fundamental interspecies difference in spinal cord repair mechanisms is associated with superior recovery in primates compared to rats after a lateral hemisection SCI. Direct motor cortex projections to motoneurons that are both numerous (37) and functionally important (36) to produce skilled movements in primates were all eliminated in injured monkeys. We thus propose that after injury, residual and new indirect corticospinal tract inputs participate in the execution of movement $(28,36)$, even in basic motor tasks for which corticospinal tract inputs are not necessary in the intact state. These cortical inputs may even become essential for the recovered execution of both locomotion and skilled movements after severe central nervous system damage $(13,36,38)$.

These findings reemphasize the importance of developing therapies that target regeneration and sprouting of the corticospinal tract in humans $(5,29,39)$ and neurorehabilitation procedures to enhance and shape the contribution of the motor cortex to functional recovery $(13,34,36)$. Regeneration of the corticospinal tract is already the primary focus of various therapeutic approaches. Although rodent models remain essential for advancing these therapies, the specificity of spinal cord repair mechanisms detected in primates suggests that these developments would be more effective and relevant in nonhuman primate models, especially for the recovery of skilled movement (40).

Contrary to the general consensus, we demonstrate that primates show greater spontaneous recovery of function than do rodents after similar spinal cord damage. The generalization of these findings to other types of incomplete injuries is unclear. Nevertheless, the demonstration that the specific anatomical and functional features of the corticospinal tract contribute to explaining this pronounced interspecies difference is particularly relevant for basic neuroscience and medicine. The community has long recognized the importance of testing safety and efficacy of new therapies in primate models of SCI before clinical studies (41). However, ethical and societal hurdles related to primate experiments, together with their cost and experimental complexity, have slowed the broad implementation of this translational framework. Despite these challenges, the present results reinforce the need for continuing the development of nonhuman primate models for translational SCI research $(11,40,42)$, with the aim of improving recovery for the millions of patients affected with SCI.

\section{MATERIALS AND METHODS}

\section{Study design}

We hypothesized that the peculiar anatomy and function of the primate corticospinal tract mediate superior functional recovery in primates compared to rodents after lateralized spinal cord damage. All clinical investigations have been conducted according to the Declaration of Helsinki principles. The Swiss Federal Ethics Committee approved all aspects of the evaluations conducted in humans. All participants 
provided written informed consent. The human data contained in the database were collected over several years in multiple clinical centers in Europe. Longitudinal recordings in four Brown-Séquard syndrome patients were collected in two independent clinical centers. All surgical and experimental procedures in monkeys were carried out using the principles outlined by Laboratory Animal Care (National Institutes of Health publication no. 85-23, revised 1985) and were approved by the Institutional Animal Care and Use Committee and the Council on Accreditation of the Association for Assessment and Accreditation of Laboratory Animal Care. Nonhuman primate studies were performed over 9 years, in two research facilities, as part of experiments designed to develop therapeutic interventions. The Veterinarian Office of the Cantons of Zurich and Vaud, Switzerland approved the evaluations conducted in rats. These experiments were repeated twice. Anatomical evaluations were performed blindly, but functional evaluations were not blinded. All the subjects with complete hemisection SCI are presented in the study. All measurements were obtained using objective readouts with high-precision equipment.

\section{Experimental animal models and human subjects}

Human subjects. Recovery of upper and lower limb function was analyzed in 437 individuals who suffered a cervical SCI. All individuals were included in the European Multicenter Study about Spinal Cord Injury (EMSCI) database (www.emsci.org). For each subject, we computed an LI that quantified the asymmetry in functional deficits at 2 weeks after SCI, as described in Supplementary Materials and Methods. We monitored motor recovery in individuals who presented distinct lateralized deficits ( $\mathrm{LI} \geq 0.5$ ). Four patients (two males and two females) met this criterion. Two of the male patients suffered traumatic SCIs at the C5 and C7 levels and showed LI of 0.82 and 0.5 , respectively. The two additional patients had experienced an ischemic injury at T5 and a disc prolapse at T8, which led to LI of 0.65 and 0.5 , respectively. The patients had not suffered from any other neurological disorder. Recovery of complex motor functions was compared to the same recordings performed in a total of 33 healthy subjects.

Monkeys. A total of 20 male monkeys (Macaca mulatta) aged 5 to 18 years (mean, $9.6 \pm 4.1$ years) were studied. No effects of age were observed on any of the variables reported herein. Four of the nine monkeys studied for functional evaluations were included in previous reports $(5,11)$ and have undergone new analyses that are the subject of the present study. Two monkeys could not be tested on the treadmill after the lesion. Two monkeys received the lateral hemisection at T10 and were only used for anatomical assessments.

Rats. A total of 30 female adult rats (Lewis) were studied $(\sim 220 \mathrm{~g}$ body weight). Animals were housed individually on a 12-hour light/ dark cycle, with access to food and water ad libitum. Temperature and humidity in the animal facilities were maintained constant in accordance to Swiss regulations for animal housing.

\section{Rehabilitation}

Human patients followed a conventional rehabilitation program. Both rats and monkeys were trained to step on a treadmill and to retrieve objects with their hand three times per week for 20 min per task.

\section{Surgical procedures}

EMG and cortical electrodes were implanted before the injury, which was performed in a second surgery, as described in Supplementary Materials and Methods.

\section{Behavioral tasks}

Locomotion. Rats, monkeys, and humans were tested on a treadmill over a range of speeds. A Plexiglass enclosure was used to maintain the rats and monkeys in position while enabling kinematics recordings. Food reward was used for positive reinforcement. The more comfortable speed to obtain consistent stepping patterns over the entire course of the recovery was selected for further analysis. This corresponded to $9 \mathrm{~cm} / \mathrm{s}$ in rats, $0.47 \mathrm{~m} / \mathrm{s}$ in monkeys, and $4 \mathrm{~km} / \mathrm{h}$ in humans. To evaluate skilled locomotion, rats $(n=9)$ and human subjects $(n=4$ SCI patients; $n=4$ healthy subjects) walked along a horizontal ladder. In humans, the ladder was placed on the floor and consisted of 10 evenly distant $(43 \mathrm{~cm}$ ) plates (length, $50 \mathrm{~cm}$; width, $15 \mathrm{~cm}$; height, $3 \mathrm{~cm}$ ). In rats, the ladder was made of 10 evenly positioned $(10 \mathrm{~cm})$ circular rungs (1-cm diameter) located $10 \mathrm{~cm}$ above the floor. Ten successive trials along the ladder were analyzed.

Hand function. Rats and monkeys were trained over several subsequent sessions to retrieve food rewards from a stick (10) until they reached a stable performance. Rats were presented with a piece of chocolate, whereas monkeys retrieved apples. The diameter of the piece of chocolate was $2 \mathrm{~mm}$, whereas the apples were sliced into eight equal pieces. Human subjects retrieved a piece of plastic tubing with a similar shape as apple slices ( $5 \mathrm{~cm}, 2$-cm diameter). Monkeys and human subjects were seated. Rats were standing quadrupedally. The subjects were given 10 trials, and the number of successful food retrievals was recorded for each session.

Time points. Recordings were made before the lesion in both rats and monkeys and at regular intervals after lesion until 2 or 6 months after injury, respectively. At this stage, both rats and monkeys had reached a plateau, that is, when no or limited improvements occurred $(5,25)$. To compare rats, monkeys, and humans at comparable time points, we focused the analysis on three salient time points: before lesion and at an early and chronic stage after the lesion. Early corresponded to the time when the subjects could locomote on the treadmill despite paralysis of the ipsilesional limbs and could reach the food reward with the ipsilesional limb but failed to retrieve the item. In humans, recordings at chronic time points were obtained between 8 and 12 months after SCI.

\section{Data acquisition and analysis}

Kinematics and electromyographic recordings. Bilateral leg or unilateral arm kinematics were recorded using 12 infrared motion capture cameras $(200 \mathrm{~Hz}$, Vicon) in rats and humans, and $4 \mathrm{TV}$ cameras (100 Hz, Basler Vision Technologies) for monkeys, as described in Supplementary Materials and Methods.

Electrophysiological recordings. In rats, access of the motor cortex to leg motoneurons was tested under fully awake conditions while the animals were suspended in a harness. A train of four stimuli (0.2 ms, 10-ms pulse length, $300 \mathrm{~Hz}, 0.5$ to $1.2 \mathrm{~mA}$ ) was delivered through the chronically implanted cortical electrode over the left motor cortex, and motor-evoked responses were recorded in the right tibialis anterior. The same rats were tested before the lesion and at early and chronic time point after the lesion. Stimulation bouts were separated by $90 \mathrm{~s}$. The amplitude and latency of motor-evoked responses were measured as previously described (13). In humans, a round coil was positioned over the location eliciting the largest possible responses in the leg muscles after transcranial magnetic stimulation. After positioning the coil, a recruitment curve was performed until reaching $100 \%$ of the output. In both rats and humans, peak-to-peak amplitudes and latencies of the evoked responses were computed from EMG 
recordings from the left and right tibialis anterior muscles. In humans, the latencies of the motor responses were normalized to the height of the subject (43).

Gait data analysis. Ten step cycles were extracted from a continuous bout of stepping on the treadmill for each condition and subject. We computed 101 parameters quantifying gait and kinematics features (table S1) for each limb and gait cycle according to published methods $(5,13,25)$. Details are in Supplementary Materials and Methods.

Hand function data analysis. For each species and time point kinematics and EMG, data were extracted from five representative reaching movements. The trajectory of the hand was segregated into the different phases of the movement: start, reach, grasp, retrieval, and end. To compute the consistency of limb endpoint trajectories, we applied a PC analysis on the 3D coordinates of the hand marker during the reach phases of all trials. The consistency of limb endpoint trajectories was calculated as the percent of variance explained by the first PC. The degree of co-contraction between proximal and distal pairs of antagonist muscles was measured as the percentage of overlapping area between two EMG traces. EMG signals were rectified and filtered with a fourth-order zero-phase shift Butterworth filter with a cutoff frequency of $30 \mathrm{~Hz}$. The co-contraction index between pairs of antagonist muscles (EMG1 and EMG2) during reaching and grasping was computed as follows:

$$
\text { Co-contraction index }=2 * \frac{\int \min [\operatorname{EMG} 1(t), \operatorname{EMG} 2(t)] d t}{\int \operatorname{EMG} 1(t) d t+\int \operatorname{EMG} 2(t) d t}
$$

where EMG1 and EMG2 are the EMG activity of flexor and extensor muscles normalized to the maximum value observed during the movement, whereas min denotes the minimum between the activity of both muscles at time $t$.

\section{Statistical analysis}

All data are reported as mean values \pm SEM. Statistical evaluations were performed using one-way ANOVA for neuromorphological evaluations, one-way repeated-measures ANOVA for functional assessments, and unpaired two-tailed $t$ tests for syndromic analysis. Tukey's post hoc test was applied when appropriate. Significance was determined at the $P<0.05$ level.

\section{SUPPLEMENTARY MATERIALS}

www.sciencetranslationalmedicine.org/cgi/content/full/7/302/302ra134/DC1 Materials and Methods

Fig. S1. Clinical cases and experimental models of Brown-Séquard syndrome.

Fig. S2. Kinematics analysis uncovers similar patterns of locomotor deficits and compensation across species.

Fig. S3. Humans and monkeys, but not rats, recover hand kinematics and muscle activation patterns.

Fig. S4. Spinal cord decussating corticospinal fibers below the injury establishes synaptic contacts with neurons projecting to lumbar segments in monkeys.

Fig. S5. Increased density of corticospinal fibers above the hemisection in both rats and monkeys.

Fig. S6. Increased density of corticospinal tract fibers originating from the ipsilesional motor cortex fibers in segments below the hemisection in both rats and monkeys.

Fig. S7. Density of 5-hydroxytryptamine fibers below the hemisection remains unchanged in both monkeys and rats.

Fig. S8. The motor cortex fails to regain access to motoneurons below the hemisection in rats. Table S1. Computed gait parameters.

Movie S1. Recovery of locomotion in monkeys and humans compared to rats after a lateral hemisection of the spinal cord.

Movie S2. Recovery of hand function in monkeys and humans compared to rats after a lateral hemisection of the spinal cord.

\section{REFERENCES AND NOTES}

1. A. Curt, H. J. Van Hedel, D. Klaus, V. Dietz; EM-SCI Study Group, Recovery from a spinal cord injury: Significance of compensation, neural plasticity, and repair. J. Neurotrauma 25, 677-685 (2008).

2. J. W. Little, E. Halar, Temporal course of motor recovery after Brown-Sequard spinal cord injuries. Paraplegia 23, 39-46 (1985).

3. C. E. Brown-Sequard, Lectures on the physiology and pathology of the central nervous system and on the treatment of organic nervous affections. Lancet 2, 593-595, 659-662, 755-757, 821-823 (1868).

4. E. S. Rosenzweig, J. H. Brock, M. D. Culbertson, P. Lu, R. Moseanko, V. R. Edgerton, L. A. Havton, M. H. Tuszynski, Extensive spinal decussation and bilateral termination of cervical corticospinal projections in rhesus monkeys. J. Comp. Neurol. 513, 151-163 (2009).

5. E. S. Rosenzweig, G. Courtine, D. L. Jindrich, J. H. Brock, A. R. Ferguson, S. C. Strand, Y. S. Nout R. R. Roy, D. M. Miller, M. S. Beattie, L. A. Havton, J. C. Bresnahan, V. R. Edgerton, M. H. Tuszynski, Extensive spontaneous plasticity of corticospinal projections after primate spinal cord injury. Nat. Neurosci. 13, 1505-1510 (2010).

6. R. N. Lemon, Descending pathways in motor control. Annu. Rev. Neurosci. 31, 195-218 (2008).

7. A. R. Ferguson, K. A. Irvine, J. C. Gensel, J. L. Nielson, A. Lin, J. Ly, M. R. Segal, R. R. Ratan, J. C. Bresnahan, M. S. Beattie, Derivation of multivariate syndromic outcome metrics for consistent testing across multiple models of cervical spinal cord injury in rats. PLOS One 8, e59712 (2013).

8. L. Filli, B. Zörner, O. Weinmann, M. E. Schwab, Motor deficits and recovery in rats with unilateral spinal cord hemisection mimic the Brown-Séquard syndrome. Brain 134, 2261-2273 (2011).

9. A. Takeoka, I. Vollenweider, G. Courtine, S. Arber, Muscle spindle feedback directs locomotor recovery and circuit reorganization after spinal cord injury. Cell 159, 1626-1639 (2014).

10. L. A. Sacrey, M. Alaverdashvili, I. Q. Whishaw, Similar hand shaping in reaching-for-food (skilled reaching) in rats and humans provides evidence of homology in release, collection, and manipulation movements. Behav. Brain Res. 204, 153-161 (2009).

11. Y. S. Nout, E. S. Rosenzweig, J. H. Brock, S. C. Strand, R. Moseanko, S. Hawbecker, S. Zdunowski, J. L. Nielson, R. R. Roy, G. Courtine, A. R. Ferguson, V. R. Edgerton, M. S. Beattie, J. C. Bresnahan, M. H. Tuszynski, Animal models of neurologic disorders: A nonhuman primate model of spinal cord injury. Neurotherapeutics 9, 380-392 (2012).

12. Y. S. Nout, A. R. Ferguson, S. C. Strand, R. Moseanko, S. Hawbecker, S. Zdunowski, J. L. Nielson R. R. Roy, H. Zhong, E. S. Rosenzweig, J. H. Brock, G. Courtine, V. R. Edgerton, M. H. Tuszynski, M. S. Beattie, J. C. Bresnahan, Methods for functional assessment after C7 spinal cord hemisection in the rhesus monkey. Neurorehabil. Neural Repair 26, 556-569 (2012).

13. R. van den Brand, J. Heutschi, Q. Barraud, J. DiGiovanna, K. Bartholdi, M. Huerlimann, L. Friedli, I. Vollenweider, E. M. Moraud, S. Duis, N. Dominici, S. Micera, P. Musienko, G. Courtine, Restoring voluntary control of locomotion after paralyzing spinal cord injury. Science 336, 1182-1185 (2012).

14. J. B. Nielsen, M. A. Perez, M. Oudega, M. Enriquez-Denton, J. M. Aimonetti, Evaluation of transcranial magnetic stimulation for investigating transmission in descending motor tracts in the rat. Eur. J. Neurosci. 25, 805-814 (2007).

15. M. Linting, J. J. Meulman, P. J. Groenen, A. J. van der Koojj, Nonlinear principal components analysis: Introduction and application. Psychol. Methods 12, 336-358 (2007).

16. J. Beauparlant, R. van den Brand, Q. Barraud, L. Friedli, P. Musienko, V. Dietz, G. Courtine, Undirected compensatory plasticity contributes to neuronal dysfunction after severe spinal cord injury. Brain 136, 3347-3361 (2013).

17. G. D. Muir, I. Q. Whishaw, Complete locomotor recovery following corticospinal tract lesions: Measurement of ground reaction forces during overground locomotion in rats. Behav. Brain Res. 103, 45-53 (1999).

18. G. Courtine, R. R. Roy, J. Raven, J. Hodgson, H. McKay, H. Yang, H. Zhong, M. H. Tuszynski, V. R. Edgerton, Performance of locomotion and foot grasping following a unilateral thoracic corticospinal tract lesion in monkeys (Macaca mulatta). Brain 128, 2338-2358 (2005).

19. D. G. Lawrence, H. G. Kuypers, The functional organization of the motor system in the monkey: I. The effects of bilateral pyramidal lesions. Brain 91, 1-14 (1968).

20. P. W. Nathan, Effects on movement of surgical incisions into the human spinal cord. Brain 117, 337-346 (1994).

21. D. Barthélemy, M. J. Grey, J. B. Nielsen, L. Bouyer, Involvement of the corticospinal tract in the control of human gait. Prog. Brain Res. 192, 181-197 (2011).

22. R. N. Lemon, J. Griffiths, Comparing the function of the corticospinal system in different species: Organizational differences for motor specialization? Muscle Nerve 32, 261-279 (2005).

23. F. M. Bareyre, M. Kerschensteiner, O. Raineteau, T. C. Mettenleiter, O. Weinmann, M. E. Schwab, The injured spinal cord spontaneously forms a new intraspinal circuit in adult rats. Nat. Neurosci. 7, 269-277 (2004).

24. E. Jankowska, S. A. Edgley, How can corticospinal tract neurons contribute to ipsilateral movements? A question with implications for recovery of motor functions. Neuroscientist 12, 67-79 (2006). 
25. G. Courtine, B. Song, R. R. Roy, H. Zhong, J. E. Herrmann, Y. Ao, J. Qi, V. R. Edgerton M. V. Sofroniew, Recovery of supraspinal control of stepping via indirect propriospinal relay connections after spinal cord injury. Nat. Med. 14, 69-74 (2008).

26. M. Ballermann, K. Fouad, Spontaneous locomotor recovery in spinal cord injured rats is accompanied by anatomical plasticity of reticulospinal fibers. Eur. J. Neurosci. 23, 1988-1996 (2006).

27. B. Zörner, L. C. Bachmann, L. Filli, S. Kapitza, M. Gullo, M. Bolliger, M. L. Starkey, M. Röthlisberger, R. R. Gonzenbach, M. E. Schwab, Chasing central nervous system plasticity: The brainstem's contribution to locomotor recovery in rats with spinal cord injury. Brain 137, 1716-1732 (2014).

28. B. Zaaimi, S. A. Edgley, D. S. Soteropoulos, S. N. Baker, Changes in descending motor pathway connectivity after corticospinal tract lesion in macaque monkey. Brain 135, 2277-2289 (2012)

29. M. Oudega, M. A. Perez, Corticospinal reorganization after spinal cord injury. J. Physiol. 590, 3647-3663 (2012).

30. H. G. Kuypers, The descending pathways to the spinal cord, their anatomy and function. Prog. Brain Res. 11, 178-202 (1964).

31. P. S. Fishman, Retrograde changes in the corticospinal tract of posttraumatic paraplegics. Arch. Neurol. 44, 1082-1084 (1987).

32. N. Weidner, A. Ner, N. Salimi, M. H. Tuszynski, Spontaneous corticospinal axonal plasticity and functional recovery after adult central nervous system injury. Proc. Natl. Acad. Sci. U.S.A. 98, 3513-3518 (2001).

33. P. Freund, N. Weiskopf, J. Ashburner, K. Wolf, R. Sutter, D. R. Altmann, K. Friston, A. Thompson A. Curt, MRI investigation of the sensorimotor cortex and the corticospinal tract after acute spinal cord injury: A prospective longitudinal study. Lancet Neurol. 12, 873-881 (2013).

34. S. L. Thomas, M. A. Gorassini, Increases in corticospinal tract function by treadmill training after incomplete spinal cord injury. J. Neurophysiol. 94, 2844-2855 (2005).

35. A. Curt, M. E. Keck, V. Dietz, Functional outcome following spinal cord injury: Significance of motor-evoked potentials and ASIA scores. Arch. Phys. Med. Rehabil. 79, 81-86 (1998).

36. Y. Nishimura, H. Onoe, Y. Morichika, S. Perfiliev, H. Tsukada, T. Isa, Time-dependent central compensatory mechanisms of finger dexterity after spinal cord injury. Science 318, 1150-1155 (2007).

37. R. J. Morecraft, J. Ge, K. S. Stilwell-Morecraft, D. W. McNeal, M. A. Pizzimenti, W. G. Darling Terminal distribution of the corticospinal projection from the hand/arm region of the primary motor cortex to the cervical enlargement in rhesus monkey. J. Comp. Neurol. 521, 4205-4235 (2013).

38. A. S. Wahl, W. Omlor, J. C. Rubio, J. L. Chen, H. Zheng, A. Schröter, M. Gullo, O. Weinmann, K. Kobayashi, F. Helmchen, B. Ommer, M. E. Schwab, Neuronal repair. Asynchronous therapy restores motor control by rewiring of the rat corticospinal tract after stroke. Science $\mathbf{3 4 4}$, 1250-1255 (2014)

39. K. Liu, Y. Lu, J. K. Lee, R. Samara, R. Willenberg, I. Sears-Kraxberger, A. Tedeschi, K. K. Park, D. Jin, B. Cai, B. Xu, L. Connolly, O. Steward, B. Zheng, Z. He, PTEN deletion enhances the regenerative ability of adult corticospinal neurons. Nat. Neurosci. 13, 1075-1081 (2010).

40. G. Courtine, M. B. Bunge, J. W. Fawcett, R. G. Grossman, J. H. Kaas, R. Lemon, I. Maier, J. Martin R. J. Nudo, A. Ramon-Cueto, E. M. Rouiller, L. Schnell, T. Wannier, M. E. Schwab, V. R. Edgerton,
Can experiments in nonhuman primates expedite the translation of treatments for spinal cord injury in humans? Nat. Med. 13, 561-566 (2007).

41. B. K. Kwon, L. J. Soril, M. Bacon, M. S. Beattie, A. Blesch, J. C. Bresnahan, M. B. Bunge S. A. Dunlop, M. G. Fehlings, A. R. Ferguson, C. E. Hill, S. Karimi-Abdolrezaee, P. Lu, J. W. McDonald, H. W. Müller, M. Oudega, E. S. Rosenzweig, P. J. Reier, J. Silver, E. Sykova, X. M. Xu, J. D. Guest W. Tetzlaff, Demonstrating efficacy in preclinical studies of cellular therapies for spinal cord injury-How much is enough? Exp. Neurol. 248, 30-44 (2013).

42. P. R. Roelfsema, S. Treue, Basic neuroscience research with nonhuman primates: A small but indispensable component of biomedical research. Neuron 82, 1200-1204 (2014).

43. J. A. Petersen, B. J. Wilm, J. von Meyenburg, M. Schubert, B. Seifert, Y. Najafi, V. Dietz, S. Kollias, Chronic cervical spinal cord injury: DTI correlates with clinical and electrophysiological measures. J. Neurotrauma 29, 1556-1566 (2012).

Acknowledgments: We thank L. Qin and R. Museanko for care and behavioral training of the monkeys. Funding: Supported by the European Community's Seventh Framework Programme (CP-IP 258654, NEUWalk), the Swiss National Science Foundation (subside 31003A_146925), the International Paraplegic Foundation, the U.S. NIH (NS42291, NS049881, NS067092, NS079030, and NS09881), the U.S. Veterans Administration, the Craig H. Neilsen Foundation, Dr. Miriam and Sheldon G. Adelson Medical Research Foundation, and Russian Science Foundation (RSF grant no.14-15-00788, surgical development of cervical hemisection). Author contributions: L.F., J.B., M.S., A.C., and G.C. collected and analyzed the data in humans. M.S.B., J.C.B., L.F., S.C.S., and G.C. designed and supervised testing of motor and behavior function in monkeys. L.F., E.S.R., M.S., L.A., Y.N.-L., S.Z., S.C.S., R.v.d.B., and G.C. collected data in animal models. A.R.F., J.L.N., L.F., L.A., N.D. E.S.R., Q.B., M.S., R.v.d.B., L.A.H., M.H.T., and G.C. analyzed data in animal models. L.F., E.S.R., P.M., H.Z., R.R.R., J.C.B., Y.N.-L., M.H.T., and G.C. performed surgeries. L.F., Q.B., E.S.R., and L.A.H. conducted staining and microscopy experiments. J.L.N., L.F., and A.R.F. performed statistical analyses. M.S.B., J.C.B., E.B., J.B., V.R.E., A.R.F., A.C., M.H.T., and G.C. conceived and supervised the studies. L.F., Q.B., and G.C. prepared the figures. G.C. coordinated data integration and wrote the manuscript, and all the authors contributed to its editing. Competing interests: The authors declare that they have no competing interests. Data and materials availability: Contact A.C. for information regarding access to the $\mathrm{EMSCl}$ database. The institutions affiliated with $\mathrm{EMSCl}$ are listed in Supplementary Materials.

Submitted 6 February 2015

Accepted 5 August 2015

Published 26 August 2015

10.1126/scitransImed.aac5811

Citation: L. Friedli, E. S. Rosenzweig, Q. Barraud, M. Schubert, N. Dominici, L. Awai, J. L. Nielson, P. Musienko, Y. Nout-Lomas, H. Zhong, S. Zdunowski, R. R. Roy, S. C. Strand, R. van den Brand, L. A. Havton, M. S. Beattie, J. C. Bresnahan, E. Bézard, J. Bloch, V. R. Edgerton, A. R. Ferguson, A. Curt, M. H. Tuszynski, G. Courtine, Pronounced species divergence in corticospinal tract reorganization and functional recovery after lateralized spinal cord injury favors primates. Sci. Transl. Med. 7, 302ra134 (2015). 


\section{Species-specific recovery}

Despite decades of research and success in rodent models, there are no therapies that repair the human spinal cord. Friedli et al. looked at the reorganization and function of the corticospinal tract after spinal cord injury (SCI) in rats, monkeys, and humans. In humans with lateralized SCI (affecting only one side of the spinal cord), there was greater recovery in motor function than those with more symmetric injuries; this recovery was mirrored in monkeys with a similar SCl, but not in rats. The authors looked into why such a species divergence exists, and revealed that monkeys had a greater number of bilateral axonal projections that sprouted into denervated spinal segments below the injury, whereas rats had interrupted projections and near-complete depletion of corticospinal fibers. Thus, monkeys and humans have the potential for synaptic reorganization above and below the lesion, and this corticospinal tract reorganization correlates with functional recovery. The authors suggest that primate models should be considered more frequently for research aimed at SCI repair and therapeutics, but acknowledge the importance of rodent models in the field. Furthermore, because the degree of laterality correlates with a positive outcome, the authors suggest that it be factored into clinical trial design.

A complete electronic version of this article and other services, including high-resolution figures, can be found at: /content/7/302/302ra134.full.html

Supplementary Material can be found in the online version of this article at: /content/suppl/2015/08/24/7.302.302ra134.DC1.html

Related Resources for this article can be found online at: http://stm.sciencemag.org/content/scitransmed/6/256/256ra137.full.html http://stm.sciencemag.org/content/scitransmed/5/208/208ra146.full.html http://www.sciencemag.org/content/sci/348/6232/347.full.html

Information about obtaining reprints of this article or about obtaining permission to reproduce this article in whole or in part can be found at:

http://www.sciencemag.org/about/permissions.dtl 